\title{
Avaliando Compreensão de Sinais da Libras em Escolares Surdos do Ensino Fundamental ${ }^{1}$
}

\author{
Fernando C. Capovilla \\ Universidade de São Paulo \\ Alessandra G. S. Capovilla \\ Universidade de São Francisco \\ Keila Q. Viggiano \\ Márcia C. P. R. Bidá \\ Universidade de São Paulo
}

\begin{abstract}
RESUMO
A educação brasileira carece de instrumentos para avaliar o desenvolvimento lingüísitco da população escolar surda. O Teste de Vocabulário Receptivo de Sinais da Libras (TVRSL1) avalia a compreensão de sinais da Libras. Foi aplicado com sinalização ao vivo coletivamente na sala de aula em duas sessões de 45 minutos a 505 estudantes de 6 a 41 anos, a maioria sinalizadora com surdez profunda pré-lingual e perilingual, de $1^{\text {a. }}$ série do ensino fundamental até $1^{\text {a. }}$ do médio, de seis escolas do estado de São Paulo, quatro Escolas Municipais de Educação Especial e duas particulares. Foi normatizado de $1^{\text {a. }}$ a $8^{\text {a. }}$ série e validado por comparação com dez testes normatizados de desenvolvimento de compreensão de sinais, leitura e escrita. Resultados revelaram aumento significativo da compreensão de sinais de $1^{\text {a. a }} 8^{\text {a. }}$ série, e maiores correlações com nomeação de sinais por escolha de palavras e por escrita livre do que com nomeação de figuras por escolha de palavras e por escrita livre, com competência de leitura de palavras ou com compreensão de leitura de sentenças. Análise de itens permitiu obter a versão reordenada, com grau de dificuldade crescente, e a abreviada com apenas 66 itens para permitir avaliação ainda mais rápida e eficaz.

Palavras-chave: linguagem; vocabulário; Libras.
\end{abstract}

\section{ABSTRACT \\ Assessing Libras Sign Comprehension by Deaf Children in Elementary School}

The Brazilian school system is in need of assessment tools to evaluate language development of its deaf students . Libras Receptive Sign Vocabulary Test (LRSVT) assesses sign comprehension in Brazilian Sign Language. It was applied collectively with live signing in a population 505 students aged 6 to 41 years in 1st to 9th grades. Most of the signers were with profound prelingual or perilingual deafness, enrolled in six Sao Paulo State schools, four special public and two private schools . LRSVT was standardized from the $1^{\text {st }}$ to the $8^{\text {th }}$ grades and validated by comparison with ten standardized tests for assessing comprehension for word and sentence reading, picture and sign naming through matching-to-sample and spelling. Results revealed a significant increase in receptive sign vocabulary from the $1^{\text {st }}$ to the $8^{\text {th }}$ grade. Correlation with sign naming by matching-to-sample and spelling was greater than those with picture naming by matching-to-sample and spelling, word and sentence reading comprehension. Item analysis permitted obtaining a reordered version with increasing difficulty level, and a short version for faster assessment.

Keywords: language; vocabulary; Libras.

\section{INTRODUÇÃO}

A língua de sinais da comunidade surda brasileira é a Língua de Sinais Brasileira, também chamada Libras (Capovilla \& Raphael, 2001; Capovilla e cols., 2003). Trata-se de uma língua visoespacial com princípios formacionais únicos. Em abril de 2002, a Presidência da República sancionou a Lei Federal 10.436, decretada pelo Congresso Nacional, que oficializa a Libras em todo o território nacional (Ministério da Educação, 2002). Essa lei reconhece a Libras como meio legal de comunicação e expressão das comunidades de pessoas surdas do Brasil, determina que o poder público se empenhe no apoio e difusão do uso da Libras como meio de comunicação objetiva, que as instituições públicas prestem atendimento aos surdos em Libras e que os sistemas educacionais federal, estaduais e municipais incluam o ensino da Libras como parte dos Parâmetros Curriculares Nacionais nos cursos de formação de Educação Especial, Fonoaudiologia e Magistério, nos Ensinos Médio e Superior. Contudo, o cumprimento dessa lei vem sendo impedido pela carência de materiais de avaliação e ensino desenvolvi- 
dos especialmente para a criança surda brasileira. Mais precisamente, pela carência de materiais instrucionais que permitam ministrar o ensino bilíngüe baseado na Libras e de instrumentos de avaliação que permitam acompanhar o desenvolvimento da linguagem escrita e de sinais. De fato, a ciência e a educação brasileiras carecem de instrumentos para acompanhar o desenvolvimento de importantes competências lingüísticas na população escolar surda, como a competência de leitura em Português, a competência de escrita em Português e o vocabulário de sinais da Libras, dentre tantas outras, o que dificulta comparar a eficácia relativa de diferentes métodos e procedimentos disponíveis para a escolarização e o ensino de leitura e escrita. Este artigo descreve o Teste de Vocabulário Receptivo de Sinais da Libras (TVRSL1), um instrumento normatizado e validado para avaliar a compreensão de sinais da Libras por parte de escolares do ensino fundamental, que pode contribuir para elucidar as complexas relações entre aquisição de Libras e de Português, mapear o desenvolvimento da linguagem em surdos sob diferentes condições de ensino, e prevenir atraso de linguagem.

$\mathrm{O}$ atraso de linguagem é o problema de desenvolvimento mais comum em pré-escolares (Bax, Hart \& Jenkins, 1980) e está fortemente correlacionado com posteriores problemas de aprendizagem (Aram, Ekelman \& Nation, 1984). Pode ser identificado já a partir dos dois anos de idade, avaliando o número de itens lexicais que a criança emite (i.e., vocabulário expressivo) e compreende (i.e., vocabulário receptivo). Tais itens lexicais consistem em palavras faladas para a ouvinte e em sinais para a surda. Estudos conduzidos em crianças ouvintes e com desenvolvimento lingüístico normal sugerem que, aos dois anos de idade, as crianças devem apresentar um vocabulário expressivo de pelo menos cinqüenta palavras e ser capazes de formar combinações de duas ou três palavras (Coplan, Gleason, Ryan \& Williams, 1982; Resnick, Allen \& Rapin, 1984). Tem sido demonstrado que cerca de metade das crianças identificadas como tendo atraso específico de linguagem expressiva entre 24 e 30 meses tendem a continuar apresentando severo atraso de fala entre três e quatro anos de idade (Rescorla, 1989). É clara a necessidade de desenvolver e validar instrumentos para identificar precocemente possíveis atrasos de linguagem em crianças já a partir dos dois anos de idade, de modo a oferecer intervenção precoce capaz de reverter o atraso de linguagem oral e prevenir dificuldades de aquisição de leitura e escrita.

$\mathrm{O}$ vocabulário receptivo é requisito para recepção e processamento de informação. Isso vale tanto para o vocabulário receptivo auditivo (i.e., de palavras ouvi- das) da criança ouvinte quanto para o vocabulário receptivo visual (i.e., de sinais observados) da criança surda. A extensão do vocabulário receptivo é uma das mais importantes medidas da habilidade intelectual. De fato, há pelo menos cinqüenta anos já havia sido demonstrado que provas de vocabulário receptivo fornecem o melhor índice individual de aproveitamento escolar (Dale \& Reichert, 1957). Além disso, também tem sido demonstrado que os subtestes de vocabulário são os que mais contribuem para os escores das provas de inteligência geral (Elliott, 1983).

Em crianças ouvintes com desenvolvimento normal, a aquisição do vocabulário receptivo auditivo (i.e., do léxico fonológico) tende a ser fortemente afetada pelas habilidades cognitivas, sendo que quanto maior a habilidade de inferência de uma criança, tanto maior tenderá a ser seu vocabulário receptivo auditivo, ou seja, o número de palavras faladas que ela se torna capaz de compreender. Por outro lado, em crianças surdas ou com deficiência auditiva, filhas de pais ouvintes, a aquisição do vocabulário receptivo auditivo e de outras habilidades lingüísticas relacionadas à linguagem oral tende a ficar defasada em relação ao desenvolvimento cognitivo. Ou seja, apesar de boas habilidades de inferência, a criança tende a apresentar atraso no desenvolvimento das habilidades de linguagem oral. Em crianças com perda auditiva profunda ou severa pré-lingual ou perilingual, incapazes de, mesmo com o auxílio de próteses auditivas, obter o desempenho auditivo necessário ao desenvolvimento da linguagem oral, a imersão, desde a mais tenra idade, em uma comunidade surda sinalizadora é uma das melhores estratégias para prevenir atrasos no desenvolvimento lingüístico e cognitivo.

Enquanto a relação entre inteligência e o subseqüiente desenvolvimento de habilidades lingüísticas oro-aurais tende a ser razoavelmente contínua para as crianças ouvintes, ela tende a ser descontínua para as crianças surdas filhas de pais ouvintes. Lederberg e Spencer (2001) fizeram uma revisão bibliográfica sobre o desenvolvimento de vocabulário receptivo auditivo em surdos e ouvintes na faixa de 8 meses a 20 anos de idade. O estudo contrasta o desenvolvimento de vocabulário em crianças surdas e ouvintes, com especial atenção a características como: (1) O número de palavras que a criança conhece e a natureza dessas palavras; (2) A taxa de crescimento do vocabulário; e (3) Os processos ou estratégias de aprendizagem de palavras que as crianças usam para facilitar e guiar a aprendizagem de novas palavras. Os resultados revelaram que, para crianças surdas filhas de pais ouvintes, a perda auditiva produz atraso no desenvolvimento do vocabulário, tal como refletido em léxicos menores, taxas mais baixas de aquisição de palavras 
novas e menor amplitude de contextos capazes de promover aprendizagem de palavras. A pesquisa sugere que, apesar de seu ritmo mais lento de aquisição, as crianças com surdez e deficiência auditiva acabam eventualmente adquirindo estratégias compensatórias que auxiliam a acelerar a aprendizagem de palavras.

No Brasil, resultados semelhantes quanto ao efeito da perda auditiva sobre o vocabulário de filhos surdos de ouvintes foram documentados por Portugal e Capovilla (2002), que demonstraram os efeitos empobrecedores da perda auditiva sobre o vocabulário, a consciência fonológica e o desempenho escolar de primeiranistas do ensino fundamental. Usando a versão brasileira normatizada e validada do Peabody Picture Vocabulary Test (Dunn, Dunn, Capovilla \& Capovilla, 2005a, 2005b), esse estudo revelou que, independentemente da inteligência não-verbal, quanto maior o grau de perda auditiva, tanto menores o vocabulário receptivo auditivo, a consciência fonológica, a discriminação auditiva e a nota escolar, e tanto maior a incidência de dificuldades articulatórias e de trocas e omissões articulatórias.

O vocabulário receptivo está fortemente correlacionado com a habilidade de leitura e com o quociente de inteligência (Eysenck \& Keane, 1990). A correlação com a inteligência geral decorre do fato de que a aquisição do vocabulário requer o uso de informações contextuais para fazer inferências plausíveis sobre o significado de palavras desconhecidas. Um exemplo de como inferências são empregadas para eduzir ou depreender o significado de palavras desconhecidas a partir do contexto frasal em que aparecem é fornecido por Sternberg (1985). Numa sentença como "Ao amanhecer, o blen surgiu radiante no horizonte", o significado de blen (i.e., sol) pode ser claramente deduzido a partir do contexto. Esse mesmo tipo de processamento ativo de construção de inferências que caracteriza a aquisição de vocabulário também caracteriza a compreensão auditiva de enunciados e a compreensão de leitura de textos. Daí as elevadas intercorrelações envolvendo vocabulário receptivo, compreensão auditiva de enunciados, e compreensão de leitura de textos.

Portanto, o conhecimento do significado das palavras e o desenvolvimento de conceitos são essenciais para permitir a compreensão auditiva e de leitura, de modo que a falta de conhecimento relevante reduz severamente a compreensão (Anderson \& Freebody, 1983; Kame'enui, Carnine \& Freshi, 1982; Marks, Doctorow \& Wittrock, 1974). De fato, Mezynski (1983) e Stahl e Fairbanks (1986) descobriram que o desempenho de compreensão pode ser melhorado por meio do treinamento de desenvolvimento de conceitos e palavras, e que programas voltados para a expansão do vocabulário tendem a favorecer o desenvolvimento do raciocínio inferencial e da compreensão de leitura. Por conseguinte, McKeon, Beck, Omanson e Pople (1985) demonstraram que boa parte do efeito de procedimentos que buscam aumentar a compreensão de leitura em leitores fracos por meio da expansão do vocabulário deriva do desenvolvimento de habilidades de fazer inferências em sentenças em que as palavras são usadas, mais do que simplesmente da rotineira aprendizagem das palavras apresentadas juntamente com suas definições.

O fato de que programas de expansão de vocabulário de crianças ouvintes contribuem para melhorar sua compreensão de leitura por meio do desenvolvimento das habilidades inferenciais na fala interna ajuda a compreender porque se espera que programas de expansão de vocabulário de sinais em Libras devam contribuir para a compreensão de leitura do surdo por meio do desenvolvimento das habilidades inferenciais na sinalização interna (Capovilla, no prelo). Para comparar a eficácia de diferentes programas voltados à expansão do vocabulário receptivo sobre a compreensão de leitura de surdos, é muito importante dispor de testes validados e normatizados de avaliação de habilidades de leitura, bem como de vocabulário receptivo visual de sinais em Libras. O presente TVRSL pode ser um importante primeiro passo também neste sentido.

\section{OBJETIVOS}

Este estudo objetiva apresentar a versão original do Teste de Vocabulário Receptivo de Sinais da Libras (TVRSL1) e descrever o desenvolvimento da compreensão de sinais da Libras em 505 estudantes surdos da $1^{\text {a. }}$ série do ensino fundamental até a $1^{\text {a. }}$ série do ensino médio, avaliando a sensibilidade do TVRSL1 de discriminar entre as séries escolares. Além disso, o estudo tem como objetivos disponibilizar tabela de dados normativos do vocabulário receptivo visual de sinais da Libras como função de série escolar, fornecendo subsídios para a análise do desenvolvimento do vocabulário receptivo visual de sinais da Libras ao longo das séries escolares. O estudo se propõe, ainda, a partir da freqüência de acertos em cada item, apresentar a versão reordenada com grau crescente de dificuldade (TVRSL2) e, a partir dessa versão reordenada, selecionar a faixa crítica de 66 itens mais relevantes à discriminação de diferentes séries escolares, de modo a gerar uma versão abreviada TVRSL3 para aplicação rápida e fácil do Teste de Vocabulário Receptivo de Sinais da Libras.

\section{Participantes}

\section{METODOLOGIA}

Participaram voluntariamente deste estudo 505 crianças, jovens e adultos surdos, de 6 a 41 anos de 
idade (idade média, 13 anos e 11 meses, $\mathbf{D P}=4,66$ ), estudantes de $1^{\text {a. }}$ série do ensino fundamental até $1^{\text {a. }}$ série do ensino médio, provenientes de quatro escolas municipais de educação especial de São Paulo e de duas escolas filantrópicas do interior do estado de São Paulo. Dos 505, 400 estudavam em escolas especiais para surdos, 80 em classes regulares de escolas regulares e 25 em classes especiais. Essas últimas 105 crianças não recebiam ensino em Libras, mas apenas ensino comum em Português, e tinham pouca oportunidade de contato com a Libras e a comunidade surda.

Dos 505 estudantes, 26 não tinham série escolar definida pois pertenciam a classes especiais que seguem programas e regimes diferentes. Dos demais, 71 cursavam a $1^{\text {a. }}$ série do ensino fundamental; 73, a $2^{\text {a. }}$ série; 76 , a $3^{\text {a. }}$ série; 70 , a $4^{\text {a. }}$ série; 53 , a $5^{\text {a. }}$ série; 63 , a $6^{\text {a. }}$ série; 42 , a $7^{\text {a. }}$ série; 24 , a $8^{\text {a. }}$ série; e 7 , a $1^{\text {a. }}$ série do ensino médio. A escolaridade média desses 479 estudantes era de 4,0 anos $(\mathbf{D P}=2,17)$, ou seja, $4^{\text {a. }}$ série. Dos 505 prontuários, 498 tinham dados sobre idade, que variou de 6 a 41 anos, com média de $13 \mathrm{a} 11 \mathrm{~m}$ (i.e., 13 anos e 11 meses) ( $\mathbf{D P}=4,66)$. Dos 505, 498 tinham dados de gênero, sendo que 277 eram do gênero masculino e 221, feminino.

Dos 505 prontuários, apenas 482 tinham grau de perda auditiva mencionada, embora em sua maioria não estabelecida a partir de exames audiológicos precisos. Numa escala aproximada de severidade variando de 1 a 4 (sendo 1 = leve, $2=$ moderada, $3=$ severa, $4=$ profunda $)$, a média foi de 3,72 $(\mathbf{D P}=0,56)$. Com relação ao tipo de perda auditiva (congênita ou adquirida), dos 505 estudantes, 387 tinham algum tipo de perda declarada, sendo que, destes, 288 declararam ter perda congênita (4 casos de grau de perda ignorada, 3 casos de perda leve, 28 de perda moderada, 60 de perda severa, 193 de perda profunda) e 99 declararam ter perda adquirida ( 8 casos de perda moderada, 21 casos de perda severa e 70 de perda profunda).

Quanto ao modo de comunicação com a família em casa, dos 505 estudantes, 261 nada declararam. Os 244 restantes revelaram fazer uso dos seguintes recursos: sinais (86 casos); sinal e oralização (52); oralização (59); sinal, oralização e escrita (19); gestos (18); sinal e gesto (8); oralização e gesto (1); e fala e escrita (1). Quanto ao modo de comunicação na escola, dos 505 surdos, 301 nada declararam. Os 204 restantes revelaram que fazem uso dos seguintes recursos: sinais (105 casos); oralização (55); sinal e oralização (32); gestos (9); oralização e gesto (2); e sinal e gesto (1). Quanto ao modo de comunicação com a comunidade de amigos, dos 505 surdos, 333 nada declararam. Os 172 restantes revelaram que fazem uso dos seguintes recursos: sinais (88 casos); oralização (37); sinal e oralização (37); gestos (3); sinal e gesto (3); sinal, oralização e escrita (2); e oralização e gesto (2). Assim, considerando o modo de comunicação preferido pelos participantes em diferentes ambientes, na passagem do ambiente familiar para o escolar e, deste, para o da comunidade social, foi notada uma incidência cada vez maior da sinalização (de 86 para 88 para 105 casos, respectivamente) e incidências cada vez menores de oralização (de 59 para 55 para 37 casos, respectivamente) e de gesticulação (de 18 para 9 para 3 casos, respectivamente).

\section{Material}

Foi empregada a versão original do Teste de Vocabulário Receptivo de Sinais da Libras, TVRSL1 (Capovilla e cols., 2004a), que avalia a compreensão de sinais da Libras em escolares surdos por meio da escolha de figuras que correspondem a sinais da Libras previamente validados para a mesma população. Ele constitui uma adaptação, para a Libras, do Teste de Vocabulário por Figuras USP (TVFusp) que avalia, também por meio da escolha de figuras, a compreensão de palavras do Português em escolares ouvintes. Para assegurar a validação desses sinais para essa população escolar surda, os sinais foram extraídos do "Dicionário enciclopédico ilustrado trilíngüe da Língua de Sinais Brasileira" (Capovilla \& Raphael, 2001), que traz a chancela da Federação Nacional de Educação e Integração de Surdos (Feneis), uma vez que resultou de cinco anos de pesquisas intensivas em conjunção com professores informantes surdos credenciados da Feneis e de revisão sistemática e cuidadosa ao longo de todo o sexto ano conduzida pela maior autoridade em Libras: a Coordenadoria de Ensino de Libras da Feneis.

No TVFusp para ouvintes, a cada item o avaliador pronuncia uma palavra e os examinandos escolhem, dentre quatro figuras alternativas, a que corresponde à palavra falada pelo avaliador. Evidências de validade e fidedignidade são descritas em Capovilla, Machalous e Capovilla (2003) e Capovilla, Gütschow e Capovilla (2003). Na adaptação original para surdos, o TVRSL avalia a compreensão de sinais da Libras, sendo que, a cada item, em vez de apresentar uma palavra falada em Português, o examinador apresenta um sinal da Libras, e os examinandos escolhem, dentre quatro figuras alternativas, a que melhor corresponde ao sinal mostrado ou articulado pelo avaliador.

O TVRSL produz um escore geral do vocabulário receptivo visual de sinais da Libras que indica quão bem o examinando surdo compreende tais sinais. Assim, permite acompanhar o desenvolvimento do vocabulário receptivo visual em Libras como função de 
diferentes abordagens educacionais e avaliar o impacto desse vocabulário sobre o desempenho escolar e a compreensão de leitura. Como o TVFusp, o TVRSL também contém 139 itens. O teste pode ser aplicado individualmente no contexto clínico ou coletivamente no contexto educacional em sala de aula. Além disso, pode ser aplicado com sinalização ao vivo emitida pelo avaliador ou com a exibição dos sinais padronizados com retroprojetor e transparências.

Para aplicação do TVRSL1 com sinalização ao vivo por sinalizador fluente em Libras, são usados um Guia de articulação de sinais do TVRSL1, um Caderno de resposta para escolha de figuras do TVRSL1 e um Gabarito de aferição de respostas do TVRSL1 para uso pelo examinador, além de um lápis preto e uma borracha para cada examinando surdo, conforme descrito na seção a seguir. A Figura 1 ilustra um dos itens do TVRSL1. Cada item é composto de um sinal da Libras acima e de quatro figuras alternativas de escolha, abaixo. A tarefa do examinando é simplesmente assinalar a figura que corresponde ao sinal acima. Nesse caso, basta que o examinador distribua a cada examinando um exemplar do Caderno completo de aplicação com sinais e figuras de escolha. Finalmente, também foram empregados um retroprojetor e transparências das folhas de aplicação para manter toda a classe concentrada e reduzir possibilidade de os alunos surdos se perderem durante a aplicação.

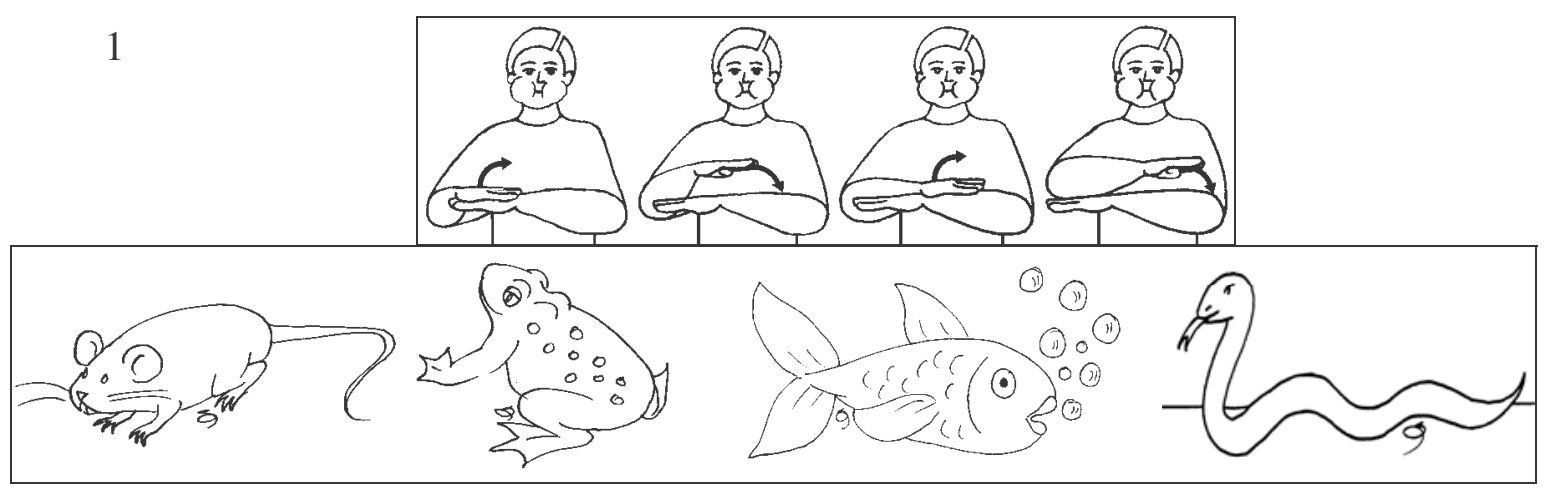

Figura 1: Amostra de um item do Caderno completo de aplicação com sinais e figuras de escolha do TVRSL1. Cada item contém o sinal da Libras acima (no caso, sinal SAPO), e quatro figuras alternativas de escolha, abaixo. Todos os sinais da Libras usados no TVRSL encontram-se documentados em Capovilla e Raphael (2001).

\section{Procedimento}

Todos os 505 estudantes das seis escolas foram avaliados em situação coletiva em sala de aula. Um único examinador fluente em Libras administrou o teste. O TVRSL foi aplicado coletivamente em sala de aula, em 90 minutos, divididos em duas sessões de 45 minutos cada uma, com articulação ao vivo dos 139 sinais pelo examinador orientado por um guia de aplicação, e coleta de dados por meio de um caderno de figuras, contendo quatro figuras para cada um dos 139 sinais apresentados pelo examinador. Previamente à aplicação, o examinador havia dividido ao meio o Caderno de figuras para aplicação do TVRSL1, reservando a primeira metade para a primeira sessão e a segunda metade para a segunda sessão. Antes de cada sessão, o examinador distribuía a metade do Caderno de figuras para aplicação do TVRSL1, um lápis preto e uma borracha para cada examinando surdo. Durante as sessões, para assegurar-se de que os sinais fossem articulados precisamente do mesmo modo, o examinador fazia uso do Guia para aplicação de sinais do TVRSL1. Ao mesmo tempo, para assegurar que os alunos não se perdessem no caderno, ele projetava as quatro alternativas de escolha na tela por meio de retroprojetor e transparências, e instruía os examinandos a escolher e assinalar uma das quatro figuras (i.e., aquela que melhor correspondesse ao significado do sinal) em seus cadernos.

\section{RESULTADOS}

Anova do escore total na versão original do Teste de Vocabulário Receptivo de Sinais da Libras (TVRSL1) como função da série escolar de 461 estudantes desde a $1^{\mathrm{a}}$ série do ensino fundamental até a $1^{\mathrm{a}}$ série do ensino médio revelou efeito significativo da série, $\mathbf{F}(8,452)=21,16, \mathbf{p}<0,000, \mathbf{r}=0,52, \mathbf{r}^{2}=$ $0,27, \mathbf{N}=461$. O escore aumentou desde a $1^{\text {a. }}$ até a $8^{\text {a }}$ série do ensino fundamental. A pontuação média derivada da Anova, e respectivos erros-padrão e número de participantes avaliados, foram os seguintes: na $1^{\text {a. }}$ série 91,60 pontos $(\mathbf{E P}=1,89, \mathbf{N}=70)$, na $2^{\text {a. }}$ série 97,70 pontos $(\mathbf{E P}=1,85, \mathbf{N}=73)$, na $3^{\text {a. }}$ série 102,79 pontos $(\mathbf{E P}=1,81, \mathbf{N}=76)$, na $4^{\text {a. }}$ série 107,46 pontos $(\mathbf{E P}=1,89, \mathbf{N}=70)$, na $5^{\text {a. }}$ série 115,86 pontos $(\mathbf{E P}=$ $2,19, \mathbf{N}=52)$, na $6^{\mathrm{a} .}$ série 116,62 pontos $(\mathbf{E P}=2,11, \mathbf{N}$ $=56)$, na $7^{\text {a. }}$ série 118,53 pontos $(\mathbf{E P}=2,56, \mathbf{N}=38)$, 
na $8^{\text {a. }}$ série 119,41 pontos $(\mathbf{E P}=3,36, \mathbf{N}=22)$, e na $1^{\text {a. }}$ série do ensino médio 116,75 pontos $(\mathbf{E P}=7,89, \mathbf{N}=$ 4). As análises de comparação de pares por meio do teste conservador de Bonferroni revelaram que o escore cresceu significativamente da $1^{\text {a }}$ série às demais a partir da $3^{\mathrm{a}}$ série; da $2^{\mathrm{a}}$ série às demais a partir da $4^{\mathrm{a}}$ série; da $3^{\mathrm{a}}$ série às demais a partir da $5^{\mathrm{a}}$ série; e da $4^{\mathrm{a}}$ série às $6^{\mathrm{a}}$ e $7^{\mathrm{a}}$ séries. A partir daí, não houve diferença significativa.

Ancova do escore total no TVRSL como função da série escolar, tendo como covariantes o grau de perda auditiva e a idade em anos, também revelou efeito significativo da série escolar, $\mathbf{F}(8,737)=30,93, \mathbf{p}<0,000$, $\mathbf{r}=0,56, \mathbf{r}^{2}=0,31, \mathbf{N}=747$, bem como do covariante idade em anos, $\mathbf{F}(1,737)=11,48, \mathbf{p}<0,001$. As análises de comparação de pares com o teste conservador de Bonferroni revelaram que o escore cresceu significativamente da $1^{\text {a. }}$ série a todas as demais a partir da $3^{\text {a. }}$ série; da $2^{\text {a. }}$ série às demais (exceto a $1^{\text {a. }}$ série do ensino médio) a partir da $4^{\text {a. }}$ série; da $3^{\text {a. }}$ série às demais (exceto a $1^{\text {a. }}$ série do ensino médio) a partir da $5^{\text {a. }}$ série; e da $4^{\text {a. }}$ série à $6^{\text {a. }}$ e $7^{\text {a. }}$ séries. Não houve diferença significativa a partir daí. A partir da Ancova com 439 estudantes com grau de perda auditiva conhecido, controlando o efeito da variação do grau de perda auditiva, foi obtida a tabela de pontuaçãopadrão para cada série escolar. A Tabela 1 sumaria parte desses dados.

Tabela 1. Segmento dos dados normativos do TVRSL1 da 1a. à 8a. série

\begin{tabular}{|c|c|c|c|c|c|c|c|c|}
\hline $\begin{array}{c}\mathrm{P} . \\
\text { bruta }\end{array}$ & $\begin{array}{c}\text { P. padrão } \\
1^{\mathrm{a}} \text { Série }\end{array}$ & $\begin{array}{l}\text { P. padrão } \\
2^{\mathrm{a}} \text { Série }\end{array}$ & $\begin{array}{c}\text { P. padrão } \\
3^{\mathrm{a}} \text { Série }\end{array}$ & $\begin{array}{c}\text { P. padrão } \\
4^{\mathrm{a}} \text { Série }\end{array}$ & $\begin{array}{l}\text { P. padrão } \\
5^{\mathrm{a}} \text { Série }\end{array}$ & $\begin{array}{l}\text { P. padrão } \\
6^{\mathrm{a}} \text { Série }\end{array}$ & $\begin{array}{c}\text { P. padrão } \\
7^{\mathrm{a}} \text { Série }\end{array}$ & $\begin{array}{c}\text { P. padrão } \\
8^{\mathrm{a}} \text { Série }\end{array}$ \\
\hline 97 & 105,70 & 98,36 & 95,45 & 88,81 & 82,05 & 79,63 & 78,41 & 77,84 \\
\hline 98 & 106,69 & 99,35 & 96,43 & 89,80 & 83,04 & 80,62 & 79,40 & 78,83 \\
\hline 99 & 107,68 & 100,34 & 97,42 & 90,80 & 84,03 & 81,61 & 80,40 & 79,82 \\
\hline 100 & 108,66 & 101,32 & 98,40 & 91,79 & 85,02 & 82,60 & 81,39 & 80,81 \\
\hline 101 & 109,65 & 102,31 & 99,39 & 92,78 & 86,01 & 83,59 & 82,38 & 81,81 \\
\hline 102 & 110,64 & 103,30 & 100,37 & 93,78 & 87,00 & 84,58 & 83,37 & 82,80 \\
\hline 103 & 111,63 & 104,29 & 101,36 & 94,77 & 87,99 & 85,58 & 84,37 & 83,79 \\
\hline 104 & 112,61 & 105,28 & 102,35 & 95,76 & 88,98 & 86,57 & 85,36 & 84,78 \\
\hline 105 & 113,60 & 106,27 & 103,33 & 96,75 & 89,97 & 87,56 & 86,35 & 85,77 \\
\hline $\begin{array}{c}\text { P. } \\
\text { bruta }\end{array}$ & $\begin{array}{c}\text { P. padrão } \\
1^{3} \text { Série }\end{array}$ & $\begin{array}{l}\text { P. padrão } \\
2^{\mathrm{a}} \text { Série }\end{array}$ & $\begin{array}{c}\text { P. padrão } \\
3^{\mathrm{a}} \text { Série }\end{array}$ & $\begin{array}{c}\text { P. padrão } \\
4^{3} \text { Série }\end{array}$ & $\begin{array}{c}\text { P. padrão } \\
5^{\mathrm{a}} \text { Série }\end{array}$ & $\begin{array}{c}\text { P. padrão } \\
6^{a} \text { Série }\end{array}$ & $\begin{array}{c}\text { P. padrão } \\
7^{\mathrm{a}} \text { Série }\end{array}$ & $\begin{array}{c}\text { P. padrão } \\
8^{\mathrm{a}} \text { Série }\end{array}$ \\
\hline 106 & 114,59 & 107,26 & 104,32 & 97,75 & 90,96 & 88,55 & 87,34 & 86,77 \\
\hline 107 & 115,57 & 108,25 & 105,30 & 98,74 & 91,96 & 89,54 & 88,33 & 87,76 \\
\hline 108 & 116,56 & 109,24 & 106,29 & 99,73 & 92,95 & 90,53 & 89,33 & 88,75 \\
\hline 109 & 117,55 & 110,22 & 107,27 & 100,72 & 93,94 & 91,52 & 90,32 & 89,74 \\
\hline 110 & 118,53 & 111,21 & 108,26 & 101,72 & 94,93 & 92,51 & 91,31 & 90,73 \\
\hline 111 & 119,52 & 112,20 & 109,24 & 102,71 & 95,92 & 93,51 & 92,30 & 91,73 \\
\hline 112 & 120,51 & 113,19 & 110,23 & 103,70 & 96,91 & 94,50 & 93,29 & 92,72 \\
\hline 113 & 121,49 & 114,18 & 111,22 & 104,70 & 97,90 & 95,49 & 94,29 & 93,71 \\
\hline 114 & 122,48 & 115,17 & 112,20 & 105,69 & 98,89 & 96,48 & 95,28 & 94,70 \\
\hline 115 & 123,47 & 116,16 & 113,19 & 106,68 & 99,88 & 97,47 & 96,27 & 95,69 \\
\hline 116 & 124,45 & 117,15 & 114,17 & 107,67 & 100,87 & 98,46 & 97,26 & 96,69 \\
\hline 117 & 125,44 & 118,13 & 115,16 & 108,67 & 101,86 & 99,45 & 98,25 & 97,68 \\
\hline 118 & 126,43 & 119,12 & 116,14 & 109,66 & 102,85 & 100,45 & 99,25 & 98,67 \\
\hline 119 & 127,41 & 120,11 & 117,13 & 110,65 & 103,84 & 101,44 & 100,24 & 99,66 \\
\hline 120 & 128,40 & 121,10 & 118,11 & 111,64 & 104,83 & 102,43 & 101,23 & 100,65 \\
\hline
\end{tabular}

A Tabela 2 sumaria as pontuações correspondentes aos percentis 1 (i.e., -3 DP), 2 (i.e., -2 DP), 16 (i.e., -1 DP), 50 (i.e., média), 84 (i.e., +1 DP), 98 (i.e., +2 DP), e 99 (i.e., +3 DP) para cada uma das oito séries escolares no TVRSL1, bem como os escores dos limites superior e inferior dos intervalos das cinco classificações de escores para cada série: Muito rebaixado (i.e., entre -3 DP e -2 DP), rebaixado (i.e., entre -2 DP e -1 DP), médio (i.e., entre -1 DP e +1 DP), elevado (i.e., entre +1 DP e +2 DP), e muito elevado (i.e., entre +2 DP e +3 DP). Ou seja, ela sumaria, para cada uma das oito séries escolares, as pontuações inferior e superior de cada um dos cinco intervalos que constituem as cinco faixas de classificação de escores. Conforme a tabela: Para a $4^{\text {a. }}$ série, as crianças com escore médio pontuam entre 93 e 123; as com escore rebaixado pontuam entre 78 e 92 ; as com escore muito rebaixado pontuam entre 63 e 77 ; as com escore elevado pontuam entre 124 e 138; e aquelas com escore muito elevado obtêm a pontuação máxima (i.e., 139 pontos). 
Tabela 2. Pontuações de cada série escolar no TVRSL1, e escores dos limites superior e inferior dos intervalos das cinco classificações de escores para cada série: Muito rebaixado (i.e., entre -3 DP e -2 DP), rebaixado (i.e., entre -2 DP e -1 DP), médio (i.e., entre -1 DPP e +1 DP), elevado (i.e., entre +1 DP e +2 DP), e muito elevado (i.e., entre +2 DP e +3 DP)

\begin{tabular}{|c|c|c|c|c|c|c|c|c|c|}
\hline \multirow[t]{2}{*}{ Série } & \multirow{2}{*}{$\begin{array}{c}\text { M ajustada } \\
\text { (Ancova) }\end{array}$} & $\underline{D P}$ & \multirow[t]{2}{*}{$\underline{\mathrm{N}}$} & MUIT & \multicolumn{2}{|c|}{ REBAIXADO } & \multicolumn{2}{|c|}{ ELEVADO } & \multirow{2}{*}{$\begin{array}{c}\text { MUITO } \\
\text { ELEVADO } \\
+3 \text { DP }\end{array}$} \\
\hline & & (Ancova) & & $-3 \mathrm{DP}$ & $-2 \mathrm{DP}$ & $-1 \mathrm{DP}$ & +1 DP & +2 DP & \\
\hline $1^{a}$ & 91,22 & 15,20 & 70 & 46 & 61 & 76 & 106 & 122 & 137 \\
\hline $2^{\mathrm{a} .}$ & 98,66 & 15,17 & 73 & 53 & 68 & 83 & 114 & 129 & 139 \\
\hline $3^{a .}$ & 101,62 & 15,22 & 76 & 56 & 71 & 86 & 117 & 132 & 139 \\
\hline $4^{\text {a. }}$ & 108,27 & 15,11 & 70 & 63 & 78 & 93 & 123 & 138 & 139 \\
\hline $5^{a}$ & 115,12 & 15,14 & 52 & 70 & 85 & 100 & 130 & 139 & ---- \\
\hline $6^{a}$ & 117,55 & 15,13 & 56 & 72 & 87 & 102 & 133 & 139 & ---- \\
\hline $7^{\mathrm{a} .}$ & 118,76 & 15,12 & 38 & 73 & 88 & 103 & 134 & 139 & ---- \\
\hline $8^{a}$ & 119,34 & 15,12 & 22 & 74 & 89 & 104 & 135 & 139 & ---- \\
\hline
\end{tabular}

Ao cabo da análise, cada um dos 139 itens teve sua incidência de acerto computada para uma amostra de 346 estudantes. Os itens foram, então, reordenados por incidência de acerto decrescente, para obter a reordenação por grau de dificuldade crescente. Portanto, nessa análise, a incidência de acerto de um dado sinal correspondia ao número de acertos obtidos naquele sinal. A incidência de acertos em qualquer sinal podia variar de zero (no caso de nenhum dos estudantes acertar o significado do sinal) a 346 (no caso de todos os estudantes acertarem o significado do sinal). A porcentagem de acertos num dado sinal correspondia à freqüência de acerto multiplicada por 100 e dividida pelo número de estudantes (i.e., 346). Por exemplo, como o sinal COBRA foi acertado por 289 dos 346 estudantes, sua porcentagem de acerto foi de $83,5 \%$. Tal análise de itens permitiu reordenar os 139 itens do TVRSL1, de modo a chegar à versão reordenada (TVRSL2) com graduação crescente de dificuldade, que permite estabelecer critérios de piso e teto como função da série escolar do estudante a ser avaliado e de seus desempenhos inicial e final. O estabelecimento de tais critérios de piso e teto torna possível aplicar apenas os itens do TVRSL2 que estão na faixa crítica mais relevante e discriminativa do desempenho de cada examinando, conforme a sua série escolar e o seu desempenho, resultando daí substancial economia de tempo e esforço de avaliação.

Para aumentar a praticidade do teste, foi elaborada também uma versão abreviada (TVRSL3) com apenas 66 itens selecionados a partir da versão reordenada, mais extensa, que contém os 139 itens ordenados por grau de dificuldade. A seleção dos itens foi feita para evitar a repetição de sinais com o mesmo grau de dificuldade. Ao permitir uma aplicação mais breve, o TVRSL3 evita o declínio do responder devido ao efeito deletério da fadiga de resposta. Ao mesmo tempo, ao cobrir, de modo equilibrado, toda a gama de variação de grau de dificuldade, a versão abreviada evita a descontinuidade na escala de graus de dificuldade, bem como os efeitos indesejáveis de piso (que ocorrem quando os itens do teste são, em sua maioria, difíceis demais para um determinado examinando) e de teto (que ocorrem quando os itens do teste são, em sua maioria, fáceis demais para um determinado examinando).

Os 66 itens foram selecionados a partir da versão reordenada TVRSL2, com grau de dificuldade crescente, de modo a cobrir toda a faixa de variação de porcentagem de acerto. Os resultados revelaram que, na amostra de 346 examinandos surdos, a freqüência de acertos nos 66 itens variou de 15 a 344, com média de 222,73 acertos e desvio-padrão de 83,58 . A porcentagem de acertos dos estudantes nesses 66 itens variou de $4,3 \%$ a $99,40 \%$, com média de $65,40 \%$ e desvio-padrão de 22,97. A nova versão abreviada TVRSL3 com apenas 66 itens tem uma série de vantagens: é de aplicação mais fácil e breve, em apenas uma sessão, e evita efeitos de teto e de piso. A Tabela 3 sumaria a ordem dos 66 sinais do TVRSL3 e a porcentagem de acerto obtida em cada um desses sinais no presente estudo com o TVRSL1.

Tabela 3. Versão abreviada TVRSL3 com 66 itens ordenados por dificuldade crescente, selecionados dos 139 itens da versão reordenada TVRSL2, e que cobrem toda a amplitude de variação de porcentagem de acerto (de 4,3\% a 99,40\%). Para cada sinal encontra-se sumariada a porcentagem de acertos (i.e., \%A) no estudo da versão original TVRSL1

\begin{tabular}{ccccccccc}
\hline Ordem & Sinal & \% Acerto & Ordem & Sinal & \% Acerto & Ordem & Sinal & \% Acerto \\
\hline $\mathbf{1}$ & TREM & 99,4 & $\mathbf{2 3}$ & ESCORPIÃO & 75,1 & $\mathbf{4 5}$ & MEIA & 61,6 \\
$\mathbf{2}$ & TARTARUGA & 97,4 & $\mathbf{2 4}$ & SEDE & 74,9 & $\mathbf{4 6}$ & GREVE & 60,4 \\
$\mathbf{3}$ & DISTRAÇÃO & $\mathbf{9 6 , 8}$ & $\mathbf{2 5}$ & DUPLA & 73,1 & $\mathbf{4 7}$ & DESERTO & 58,4 \\
$\mathbf{4}$ & PROCURAR & $\mathbf{9 6 , 5}$ & $\mathbf{2 6}$ & VAZIO & $\mathbf{7 2 , 8}$ & $\mathbf{4 8}$ & DIALOGAR & 55,5 \\
$\mathbf{5}$ & ENCANADOR & $\mathbf{9 5 , 1}$ & $\mathbf{2 7}$ & GRUPO & $\mathbf{7 2 , 8}$ & $\mathbf{4 9}$ & VOLÚVEL & 54,3
\end{tabular}




\begin{tabular}{|c|c|c|c|c|c|c|c|c|}
\hline 6 & TERMÔMETRO & 94,5 & 28 & VENCEDOR & 72,0 & 50 & BICAMPEÃO & 53,2 \\
\hline 7 & DESCASCAR & 93,1 & 29 & PÉ & 71,4 & 51 & ADORAR & 52,3 \\
\hline 8 & MECÂNICO & 92,2 & 30 & INOCENTE & 71,4 & 52 & SAUDADE & 52,3 \\
\hline 9 & VERTIGEM & 91,9 & 31 & ABAJUR & 70,5 & 53 & VENENOSO & 51,7 \\
\hline 10 & DESENHISTA & 88,7 & 32 & ESPANTADO & 70,2 & 54 & MARINHA & 50,9 \\
\hline 11 & AGRICULTURA & 87,0 & 33 & ANGÚSTIA & 69,1 & 55 & PROCESSO & 48,3 \\
\hline 12 & COMPRAR & 85,8 & 34 & MULTA & 68,8 & 56 & DESLEIXADO & 36,1 \\
\hline 13 & BATIZAR & 83,8 & 35 & PROJETO & 68,5 & 57 & FÍGADO & 35,0 \\
\hline 14 & COBRA & 83,5 & 36 & LUSTRAR & 68,2 & 58 & FAMOSO & 34,4 \\
\hline 15 & ACONSELHAR & 80,6 & 37 & CASTELO & 67,9 & 59 & HIERARQUIA & 29,8 \\
\hline 16 & APELIDO & 80,1 & 38 & RAMALHETE & 67,1 & 60 & EUROPA & 29,5 \\
\hline 17 & ENSINAR & 79,8 & 39 & IRA & 66,2 & 61 & OBRIGAR & 16,5 \\
\hline 18 & VIDRO & 79,2 & 40 & $\mathrm{SOB}$ & 65,9 & 62 & PULMÃO & 13,3 \\
\hline 19 & VELA & 77,2 & 41 & DICIONÁRIO & 63,6 & 63 & CIENTISTA & 9,0 \\
\hline 20 & BÚSSOLA & 77,2 & 42 & SAPATO & 63,0 & 64 & FELINO & 6,6 \\
\hline 21 & DERRAMAR & 76,6 & 43 & ETERNO & 62,7 & 65 & RAPOSA & 5,2 \\
\hline 22 & SOFÁ & 76,0 & 44 & SUBORNAR & 62,4 & 66 & ALARME & 4,3 \\
\hline
\end{tabular}

\section{DISCUSSÃO}

O Teste de Vocabulário Receptivo de Sinais da Libras (TVRSL) é parte da bateria de onze testes originais de desenvolvimento das competência de leitura, escrita e compreensão de sinais (Capovilla, no prelo) normatizada e validada para a população escolar surda do ensino fundamental ao médio, numa série de estudos conduzidos com sinalização ao vivo a uma amostra de 1.158 escolares surdos. O TVRSL contém 139 itens, cada qual composto de um sinal da Libras e quatro figuras alternativas de escolha. A tarefa consiste em escolher, dentre as quatro figuras alternativas, a que melhor corresponde ao sinal da Libras. O teste produz um escore geral do vocabulário receptivo visual de sinais, que indica quão bem o examinando surdo compreende os sinais da Libras. O TVRSL apresenta-se em três versões: A original com 139 sinais (TVRSL1), a reordenada com os mesmos 139 sinais ordenados de acordo com grau crescente de dificuldade (TVRSL2), e a abreviada com apenas 66 sinais críticos e ordenados por grau de dificuldade crescente (TVRSL3).

Neste estudo, o TVRSL1 foi aplicado com sinalização ao vivo a uma amostra de 505 crianças, jovens e adultos surdos, de 6 a 41 anos de idade, estudantes de $1^{\text {a. }}$ série do ensino fundamental até a $1^{\text {a. }}$ série do ensino médio. Devido às limitações de espaço, as tabelas de dados normativos por série escolar para aplicação com sinalização ao vivo devem ser encontradas em um estudo paralelo (Capovilla e cols., 2004a) que fornece, ainda, todos os materiais necessários à aplicação (e.g., os cadernos completos das três versões, o guia de articulação dos sinais, a matriz para produzir as transparências de aplicação).

Neste estudo de validação e normatização do TVRSL1, a amostra de 505 escolares obteve pontuação variando de 39 a 133 pontos, com média de
106,21 pontos $(\mathbf{D P}=17,15)$, sendo que a Ancova revelou um aumento sistemático na compreensão de sinais da Libras, desde a $1^{\text {a. }}$ até a $8^{\text {a. }}$ série do ensino fundamental (i.e., de 91,60 a 97,70 a 102,79 a 107,46 a 115,86 a 116,62 a 118,53 a 119,41 pontos, respectivamente). Assim, a pontuação cresceu de maneira monotônica, embora não tenha chegado a alcançar o teto (i.e., 139 pontos), o que sugere que a escala superior pode ser expandida para além do ensino fundamental. Com base nesses dados, o estudo normatizou o TVRSL1 por série escolar.

Num outro estudo (Capovilla e cols., 2004a) foram encontradas evidências de sua validade por comparação com os resultados em dez outros testes de desenvolvimento da linguagem de sinais e escrita, igualmente normatizados para essa população escolar surda. Conforme aquele estudo, em termos de validade por critério de correlação com outros testes, os resultados revelaram que o teste de compreensão de sinais da Libras (TVRSL1) apresentou correlações mais altas (i.e., $\mathbf{r}=0,59$ a 0,60) com o teste de nomeação de sinais por escrita e com o teste de nomeação de sinais por escolha; correlações mais baixas (i.e., $\mathbf{r}=$ $0,30$ a 0,41$)$ com o teste de nomeação de figuras por escolha, com o teste de nomeação de figuras por escrita, com o teste de competência de leitura de sentenças e com o teste de competência de leitura de palavras. Ou seja, a compreensão de sinais da Libras esteve mais correlacionada com os desempenhos de nomeação de sinais por escrita livre e por escolha do que com os desempenhos de nomeação de figuras por escrita ou escolha, ou com as competências de leitura de palavras ou de sentenças.

Além da tabela de normatização e dos dados de validação, a partir da análise do grau de dificuldade de cada um dos 139 itens, o estudo obteve, também, a versão reordenada TVRSL2, em que os 139 itens encontram-se ordenados por grau de dificuldade cres- 
cente. Finalmente, a partir da mesma análise do grau de dificuldade médio dos 139 itens, o estudo ofereceu ainda a versão abreviada TVRSL3 composta dos 66 itens mais importantes para avaliação rápida e eficaz do vocabulário receptivo de sinais. Nela foram eliminados itens que apresentavam os mesmos graus de dificuldade baixo e médio, e preservados apenas itens com graus de dificuldade diferentes capazes de cobrir toda a escala.

O TVRSL é parte de uma bateria normatizada e validada para a avaliação do desenvolvimento das competências de leitura, escrita e compreensão de sinais, cujo advento permitirá conduzir pesquisa sistemática e parametrizada para revelar quais são as abordagens e os procedimentos, os métodos e os materiais instrucionais mais indicados para diferentes parcelas da população escolar surda brasileira, dependendo de características como o tipo e o grau da perda auditiva e a idade em que essa perda auditiva ocorreu, o acesso à comunidade surda sinalizadora e ao ensino em língua de sinais e a idade em que esse acesso se deu, o acesso a diferentes procedimentos de ensino de leitura e escrita e a idade em que ele se deu, o implante coclear e a idade em que ele se deu, e assim por diante.

A política oralista de "normalização" que procura encaixar pessoas com surdez profunda ou severa prélingual ou perilingual na mesma distribuição normal da população ouvinte, comparando seu desempenho ao dos ouvintes em tarefas centradas apenas em comunicação oral-aural e em leitura e escrita do Português, vem sendo criticada pelo bilingüismo. Em território alheio, avaliados na língua dos ouvintes com instrumentos desenvolvidos por ouvintes e para ouvintes, não surpreendentemente os surdos tendem a concentrar-se no extremo inferior da distribuição de escores da população ouvinte em geral. A perspectiva médica oralista de reduzir o surdo apenas à condição de deficiente auditivo é insuficiente. É preciso conciliá-la com a perspectiva antropológica que reconhece o surdo como membro de cultura sinalizada. Só assim se poderá começar a fazer uso eficiente da língua de sinais como recurso educacional. A população surda sinalizadora tem as suas próprias normas, consideradas as particularidades da língua de sinais e de sua representação cognitiva como linguagem no indivíduo. As habilidades cognitivas e lingüísticas podem encontrar-se distribuídas na população surda de forma análoga àquela em que elas se encontram distribuídas na população ouvinte. Tomando a população surda em seu próprio direito, pode-se concebê-la, de maneira técnica e heurística, como uma população normal, em que as habilidades cognitivas e lingüísticas podem encontrar-se distribuídas de maneira normal. Daí a necessidade de instrumentos especialmente desenhados para mapear essa distribuição normal de habilidades lingüísticas, tanto em Libras quanto em Português, e para revelar os processos envolvidos em cada uma das línguas e em sua interseção, de modo a permitir a avaliação e o ensino na Libras, e o melhor uso dessa língua para a aquisição do Português escrito. São necessários instrumentos de avaliação suficientemente válidos e precisos para revelar essas normas e os processos cognitivos a elas subjacentes, bem como procedimentos de intervenção educacional suficientemente eficazes para aperfeiçoar os processos de aprendizagem, e maximizar as chances de sucesso da população surda em seu mundo que é, também, um mundo letrado. Ao oferecer um teste original e demonstrar seu emprego para caracterizar a distribuição normativa das habilidades de compreensão de sinais na população escolar surda do ensino fundamental de São Paulo, o presente artigo constitui um primeiro importante passo para que estudos subseqüentes possam elucidar as relações entre desenvolvimento da Libras e aquisição do Português, tanto no nível da palavra (e.g., Capovilla e cols., 2004b) quanto do texto (e.g., Capovilla e cols., 2005).

\section{REFERÊNCIAS}

Anderson, R. C. \& Freebody, P. (1983). Vocabulary knowledge. Em J. T. Guthrie (Org.), Comprehension and teaching: research reviews (pp. 77-117). Newark, DE: International Reading Association.

Aram, D.; Ekelman, B. \& Nation, J. (1984). Preschoolers with language disorders: 10 years later. Journal of Speech and Hearing Research, 27(2), 232-244.

Bax, M.; Hart, H. \& Jenkins, S. (1980). Assessment of speech and language development in the young child. Pediatrics, 66, 350354.

Capovilla, A. G. S.; Gütschow, C. R. D. \& Capovilla, F. C. (2003). Instrumentos de avaliação de habilidades cognitivas relacionadas à aquisição de leitura e escrita: Análise de validade e fidedignidade. Em A. G. S. Capovilla (Org.), Avaliação e intervenção em habilidades metafonológicas e de leitura e escrita (pp. 70-90). São Paulo, SP: Memnon.

Capovilla, A. G. S.; Machalous, N. \& Capovilla, F. C. (2003). Leitura em crianças bilíngües: Uso das rotas fonológica e lexical em Português e Alemão. Em M. R. Maluf (Org.), Metalinguagem e aquisição da escrita: pesquisa e prática da alfabetização (pp. 185-216). São Paulo: Casa do Psicólogo.

Capovilla, F. C. (no prelo). Enciclopédia da Língua de Sinais Brasileira: o mundo do surdo em Libras, vol. 19: Compêndio de avaliação do desenvolvimento de linguagem de sinais, leitura e escrita em estudantes surdos do ensino fundamental ao médio. São Paulo: Edusp, Imprensa Oficial do Estado de São Paulo.

Capovilla, F. C.; Duduchi, M.; Raphael, W. D.; Luz, R. D.; Rozados, D.; Capovilla, A. G. S. \& Macedo, E. C. (2003). Brazilian sign language lexicography and technology: Dictionary, digital encyclopedia, chereme-based sign-retrieval and quadriplegic deaf communication systems. Sign Language Studies, 3(4), 393-430. 
Capovilla, F. C. \& Raphael, W. D. (2001). Dicionário enciclopédico ilustrado trilíngüe da língua de sinais brasileira (2 $2^{\text {a. }}$ ed., Vols. 1 e 2). São Paulo: Edusp e Imprensa Oficial.

Capovilla, F. C.; Viggiano, K. Q.; Capovilla, A. G. S.; Raphael, W. D.; Bidá, M. R. \& Mauricio, A. C. (2004a). Como avaliar o desenvolvimento da compreensão de sinais da Libras em surdos de $1^{\mathrm{a}}$ a $8^{\mathrm{a}}$. série do ensino fundamental: Versão 1.1 do Teste de Vocabulário Receptivo de Sinais da Libras, validada e normatizada para aplicação com sinalização ao vivo. Em F. Capovilla \& W. Raphael (Orgs.), Enciclopédia da língua de sinais brasileira: o mundo do surdo em Libras, Vol. 2 (pp. 285-827). São Paulo: Edusp e Imprensa Oficial do Estado de São Paulo.

Capovilla, F. C.; Viggiano, K.; Capovilla, A. G. S.; Raphael, W.; Mauricio, A. \& Bidá, M. (2004b). Como avaliar o desenvolvimento da competência de leitura de palavras em surdos do ensino fundamental ao médio, e analisar processos de reconhecimento e decodificação: teste de competência de leitura de palavras. Em F. Capovilla \& W. Raphael (Orgs.), Enciclopédia da língua de sinais brasileira: o mundo do surdo em libras, Vol. 1 (pp. 297-680) São Paulo, SP: Edusp e Imprensa Oficial do Estado de São Paulo.

Capovilla, F. C.; Viggiano, K.; Capovilla, A. G. S.; Raphael, W.; Bidá, M.; Neves, M. \& Mauricio, A. (2005). Como avaliar o desenvolvimento da compreensão de leitura de sentenças em surdos do ensino fundamental ao médio, e analisar processamento sintático para extração de significado: Versão original validada e normatizada do Teste de Competência de Leitura de Sentenças. Em F. C. Capovilla \& W. D. Raphael (Orgs.), Enciclopédia da língua de sinais brasileira: o mundo do surdo em Libras, Vol. 3 (pp. 405-857). São Paulo, SP: Edusp.

Coplan, J.; Gleason, J. R.; Ryan, R. \& Williams, M. L. (1982). Validation of an early language milestone scale in a high-risk population. Pediatrics, 70, 677-683.

Dale, E. \& Reichert, D. (1957). Bibliography of vocabulary studies. Columbus, OH: Ohio State University Bureau of Educational Research.

Dunn, L. M.; Dunn, D.; Capovilla, F. C. \& Capovilla, A. G. S. (2005a). Manual do examinador para o teste de vocabulário por figuras peabody: adaptação brasileira. São Paulo: Casa do Psicólogo.

Dunn, L. M.; Dunn, D.; Capovilla, F. C. \& Capovilla, A. G. S. (2005b). Teste de vocabulário por figuras peabody: adaptação brasileira. São Paulo: Casa do Psicólogo.

Elliott, C. D. (1983). The british ability scales. Manual 2: Technical and statistical information. Windsor, UK: NFER-Nelson.
Eysenck, M. W. \& Keane, M. T. (1990). Cognitive psychology: a student's handbook. New York, NY: Lawrence Erlbaum.

Kame'enui, E. J.; Carnine, D. W. \& Freshi, R. (1982). Effects of text construction and instructional procedures for teaching word meanings on comprehension of contrived passages. Reading Research Quarterly, 17(3), 367-388.

Lederberg, A. R. \& Spencer, P. E. (2001). Vocabulary development of deaf and hard of hearing children. Em M. D. Clark; M. Marschark \& M. Karchmer (Orgs.), Context, cognition, and deafness (pp. 88-112). Washington, DC: Gallaudet University Press.

Marks, C. B.; Doctorow, M. J. \& Wittrock, M. C. (1974). Word frequency and reading comprehension. Journal of Educational Research, 67(6), 259-262.

McKeon, M. G.; Beck, I. L.; Omanson, R. C. \& Pople, M. T. (1985). Some effects of the nature and frequency of vocabulary instruction on the knowledge and use of words. Reading Research Quarterly, 20(5), 522-535.

Mezynski, K. (1983). Issues concerning the acquisition of knowledge: Effects of vocabulary training on reading comprehension. Review of Educational Research, 53(2), 253-279.

Ministério da Educação (2002). Legislação específica/documentos internacionais: Leis: Lei ${ }^{\circ}$. 10.436, de 24 de abril de 2002. Obtido em junho de 2002 do World Wide Web: http://www. mec.gov.br/ seesp/legislacao.shtm.

Portugal, A. C. \& Capovilla, F. C. (2002). Triagem audiológica na $1^{\mathrm{a}}$ série: efeitos de perda auditiva sobre vocabulário, consciência fonológica, articulação da fala e nota escolar. Cadernos de Psicopedagogia, 1(2), 60-97.

Rescorla, L. (1989). The Language Development Survey: A screening tool for delayed language in toddlers. Journal of Speech and Hearing Disorders, 54(4), 587-599.

Resnick, T.; Allen, D. A. \& Rapin, I. (1984). Disorders of language development: Diagnosis and intervention. Pediatrics in Review, 6(3), 85-92.

Stahl, S. A. \& Fairbanks, M. M. (1986). The effects of vocabulary instruction: A model-based meta-analysis. Review of Educational Research, 56(1), 72-110.

Sternberg, R. J. (1985). Beyond I. Q.: a triarchic theory of human intelligence. Cambridge, MA: Cambridge University Press.

Enviado: 07/07/2004

Revisado: 10/10/2004

Aceito: $16 / 10 / 2004$

\section{Nota:}

${ }^{1}$ Esta pesquisa foi financiada pelo CNPq, com bolsa de produtividade em pesquisa para os dois primeiros autores e bolsa de mestrado para os demais três autores; pela Fapesp no Programa de Apoio a Jovens Pesquisadores para a segunda autora; bem como pela Capes no programa Proesp 2003 e pela Fundação Vitae, ambos para o primeiro autor.

\section{Sobre os autores:}

Fernando C. Capovilla: Ph.D. em Psicologia Experimental pela Temple University, EUA; Livre-Docente em Psicologia Clínica pela Universidade de São Paulo.

Endereço para correspondência: Instituto de Psicologia, Universidade de São Paulo, Av. Prof. Mello Moraes, 1.721, São Paulo, SP, 05508-900, Endereço Eletrônico: capovilla@usp.br.

Alessandra G. S. Capovilla: Doutora e Pós-Doutorada em Psicologia Experimental pela Universidade de São Paulo - Programa de PósGraduação Strictu Sensu em Psicologia, Universidade de São Francisco.

Keila Q. Viggiano: Mestra em Psicologia Experimental pela Universidade de São Paulo - Instituto de Psicologia, Universidade de São Paulo.

Márcia C. P. R. Bidá: Mestranda em Psicologia Experimental pela Universidade de São Paulo - Instituto de Psicologia, Universidade de São Paulo. 
